\title{
Effects of diuretics on postoperative AKI in patients after cardiovascular surgery under cardiopulmonary bypass
}

\section{【Introduction】}

\author{
Y. Oka', Y. Toda', Y. Sato', C. Nammba', K. Maeshima', H. Nakatsuka'
}

1 Kawasaki Medical School -Japan

Acute kidney injury (AKI) after the cardiovascular surgery is not rare. Various factors such as administration of drugs, background of the patient and cardiopulmonary bypass(CPB) are thought to be related to AKI. It is unclear whether perioperative administration of diuretics had an influence on the incidence of postoperative AKI in patients after cardiovascular procedures under CPB.

\section{【Materials and methods】}

*Retrospective observational study: January 1 to December 31 in 2016.

*Patients who underwent the cardiovascular surgery under cardiopulmonary bypass(CPB) were enrolled.

*In two groups of patients (AKI group and non AKI group), we conducted statistical analysis for background of the patient, dose of diuretics (furosemide, mannitol, carperitide and others), urine volume, blood pressure, creatinine, eGFR, CPB time, and aortic cross clamping time, etc.

*AKI was diagnosed by KDIGO criteria within 3 postoperative days, and was defined more than stage 1 .

\section{Table1 Patient characteristics}

Age (y)

Sex:male $(n, \%)$

Comorbid Disease (n)

Hypertension

Renal failure

Medications (n, \%)

Loop diuretics

Others

Preoperative CRE $(\mathrm{mg} / \mathrm{dL})$

Preoperative eGFR

$(\mathrm{ml} / \mathrm{min} / 1.73 \mathrm{~m} 2)$

Table2 Comparison AKI with Non AKI

Emergency operation ( $\mathrm{n}, \%)$
Duration of
Operation (min)
Duration of
Anesthesia (min)
Duration of CPB (min)
Duration of Ao clamp (min)
Lower body circulatory
arrest ( $\mathrm{n}, \%$ )
Postoperative urine volume
(ml/24h)
Chest drain output (ml/24h)
Intraoperative diuretic
Mannitol (ml)
Carperitide ( $\mathrm{n}, \%)$
Postoperative diuretic
Furosemide (mg/24h)
Furosemide (mg/3days)
Carperitide ( $\mathrm{n}, \%)$
Postoperative CVP
(cm H2O)
Postoperative CCI
(L/min/m2)
Blood pressure (Mean)
Intraoperative (mmHg)
Postoperative (mmHg)

AKI $(n=12)$

$76.3(69.8,82.8)$

$5(41.7 \%)$

$4(33.3 \%)$

$1(8.3 \%)$

$2(16.7 \%)$

$0(0.0 \%)$

$0.86(0.47,1.25)$

$58.1(42.1,74.1)$
$4(33.3 \%)$

$398.0(323.3,472.7)$

$473.9(395.0,552.8)$

$215.3(173.8,256.9)$

$135.8(106.5,165.2)$

$5(41.7 \%)$

$809.58(257.4,1361.8) \quad 1150.7(880.2,1421.3)$

$394.6(109.2,680.0)$

$262.5(188.7,336.3)$

$5(41.7 \%)$

$20.0(9.6,30.4)$

$158.8(96.9,220.6)$

$8(66.7 \%)$

$10.4(8.0,12.8)$

$2.5(1.9,3.0)$

$55.7(51.1,60.3)$ $62.5(57.7,67.4)$

$67.3(64.9,69.7)$

$\begin{array}{cc}\text { Non-AKI }(n=50) & P \text { value } \\ 69.6(66.5,72.8) & 0.07 \\ 29(58.0 \%) & 0.31 \\ & \\ 19(38.0 \%) & 0.76 \\ 3(6.0 \%) & 0.77 \\ & \\ 8(16.0 \%) & 0.96 \\ 9(18.0 \%) & 0.04 \\ 1.02(0.83,1.21) & 0.47 \\ 65.2(57.3,73.0) & 0.43\end{array}$

Non-AKI $(n=50) \quad P$ value

$3(6.0 \%)$

$353.5(316.9,390.2)$

$432.6(393.9,471.2)$

$164.0(143.5,184.6)$

$98.3(83.9,112.6)$

$5(10 \%)$

$369.0(229.2,508.8)$

$210.0(173.8,246.2)$

$22(44.0 \%)$

$9.0(1.5,5.7)$

$72.7(42.4,103.0)$ $29(58.0 \%)$

$9.1(7.9,10.2)$

$2.8(2.5,3.1)$

$59.6(57.4,62.0)$
0.022

0.29

0.35

0.031

0.024

0.007

0.27

0.87

0.21

0.88

0.048

0.015

0.58

0.32

0.23

0.13

0.08

\section{【Results】}

AKI group;

*no diuretics other than furosemide-spironolactone, tolvaptan preoperatively

*emergency operation

*long duration of CPB

*long duration of Ao clamp

*lower body circulatory arrest

*a larger amount of furosemide postoperatively

Multivariate analysis;

There is a significant difference in furosemide postoperatively.

\section{【Discussion】}

*risk factor of $\mathrm{AKI}$;

preoperative low renal function, low eGFR, elderly patients, female, low cardiac function, comorbid disease-hypertension, diabetes, etc. Jpn Soc Intensive Care Med. 2016;23:141-147

*the relation between perioperative diuretics and AKI; Continuous infusion of furosemide is associated with the highest rate of renal impairment, and is detrimental in the protection of renal dysfunction after cardiac surgery.

J Am Soc Nephrol, 2000;11:97-104

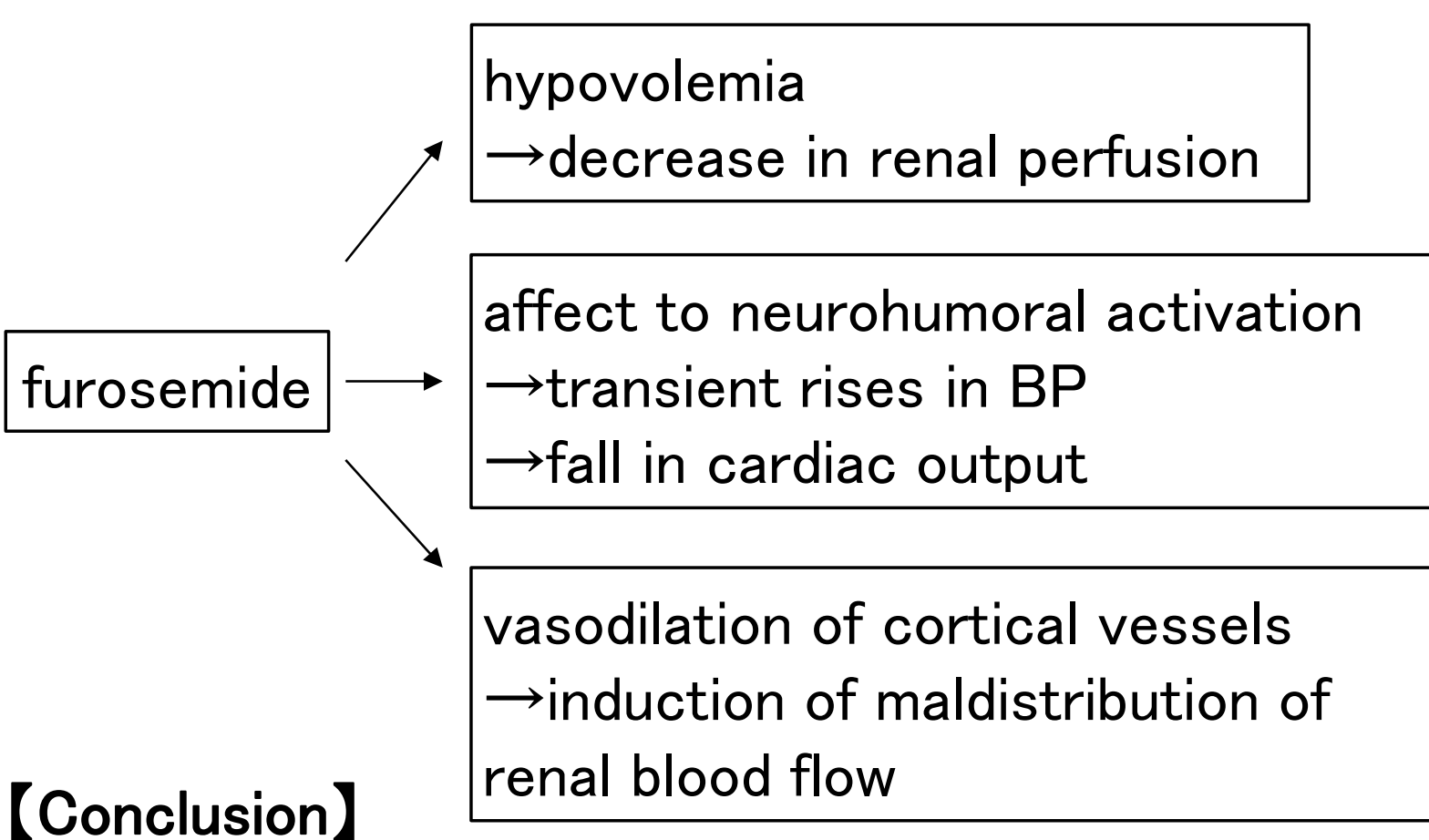

Perioperative administration of diuretics is associated with more AKI after cardiovascular surgery under cardiopulmonary bypass. 